\title{
Slot width augmentation in a slotted-wall transonic linear cascade wind tunnel
}

\author{
A. Rona* \\ Department of Engineering, University of Leicester, Leicester, LE1 7RH, UK \\ R. Paciorri ${ }^{\dagger}$ M. Geron ${ }^{\ddagger}$ \\ Department of Mechanics and Aeronautics, University of Rome "La Sapienza" \\ Via Eudossiana 18, Rome 00184, Italy \\ N.Z. Ince ${ }^{\S}$ \\ ALSTOM Power Technology Centre, Whetstone, Leicester
}

\begin{abstract}
Transonic tests in linear cascade wind tunnels can suffer from significant test section boundary interference effects in pitch. A slotted tailboard has been designed and optimised with an in-house Euler numerical method to reduce such effects. Wind tunnel measurements on an overspeed Mach 1.27 discharge from a Rolls-Royce T2 cascade, featuring strong end-wall shock-induced interference, showed a $77 \%$ reduction in the flow pitchwise periodicity error with the optimised tailboard, with respect to the baseline open-jet cascade flow. Two-dimensional Euler predictions were also cross-validated against a three-dimensional Reynolds averaged computation, to explore the three-dimensionality of the discharge.
\end{abstract}

\section{Introduction}

The use of slotted walls in transonic wind tunnels is an established practice to reduce end wall interference effects during tests. A common example of such practice is the testing of model wing planforms or model wing-fuselage assemblies for supersonic aircraft. ${ }^{1}$ Slotted walls are less commonly used in linear cascade wind tunnels, where the outer blades in the cascade may be in the proximity of the test section outer boundaries. This makes developing a slotted end wall configuration for a linear cascade tunnel interesting and challenging.

Linear cascade wind tunnels can be cost-effective tools to verify key turbine profile performance predictions from analytical and numerical design methods at an early stage of the profile development. At transonic flow regimes, the discharge from a linear cascade is affected by the cascade pitchwise boundaries that often render the discharge non-periodic across successive passages. This may lead to significant variations in profile loss coefficient from one passage to the next ${ }^{2}$ and constitutes a limiting factor for linear cascade tests. To

\footnotetext{
* Lecturer,

$\dagger$ Associate Professor,

$\ddagger$ PhD student,

$\S$ Technical Specialist.
}

restore some pitchwise periodicity in the discharge flow, perforated walls, or perforated tailboards, have been used in transonic tests at the pitchwise boundaries ${ }^{3,4}$ and a review of this technique is presented by Sieverding. ${ }^{5}$

In past work, ${ }^{3,4,6,7}$ the authors evaluated perforated and slotted walls for linear cascade tunnels. Slots were found to provide less interference with the cascade discharge with respect to perforations. Further refinements to the slotted end wall geometry have been pursued in this work, with the support of numerical models by Paciorri et al. ${ }^{6}$ and by Ince.

\section{Experimental Facility}

Slotted tailboard designs have been commissioned and tested at an intermittent blow-down linear cascade wind tunnel at the University of Leicester. A side view of the test section is shown in Fig. 1. Six $45.27 \mathrm{~mm}$ chord nozzle blades of Rolls Royce T2 profile ${ }^{8}$ are tested in a linear cascade assembly that discharges in the ambient laboratory air. There is no diffuser in this tunnel. The test section is backed by a polished stainless steel mirror and a $25.4 \mathrm{~mm}$ thick high quality schlieren glass closes the front of the test chamber. These optical surfaces are used to visualise the flow by a double-pass schlieren system. ${ }^{7,9}$ The mirror at the back of the test section is equipped with $0.5 \mathrm{~mm}$ diameter static pressure holes from which measurements of the wall static pressure in the test section are made that are synchronised with the schlieren records. ${ }^{7}$

Tests have been conducted at a settling chamber to laboratory ambient absolute pressure ratio $p_{0} / p_{a}=0.3728$. The tests used two slotted tailboards of void ratios $47 \%$ and $15 \%$, whose geometries are detailed in Fig. 2(b) and (c). The tailboards are made from $2 \mathrm{~mm}$ thick steel shims and are supported on a chassis that secures them to the test section. The two shims interchange on the same chassis, which is detailed in Fig. 2(a). This chassis is $219 \mathrm{~mm}$ long and $50 \mathrm{~mm}$ wide, spanning the test section from side to side once assembled in the cascade tunnel. The leading edge of the chassis is secured to the trailing edge of blade 0 via a brass hinge that 


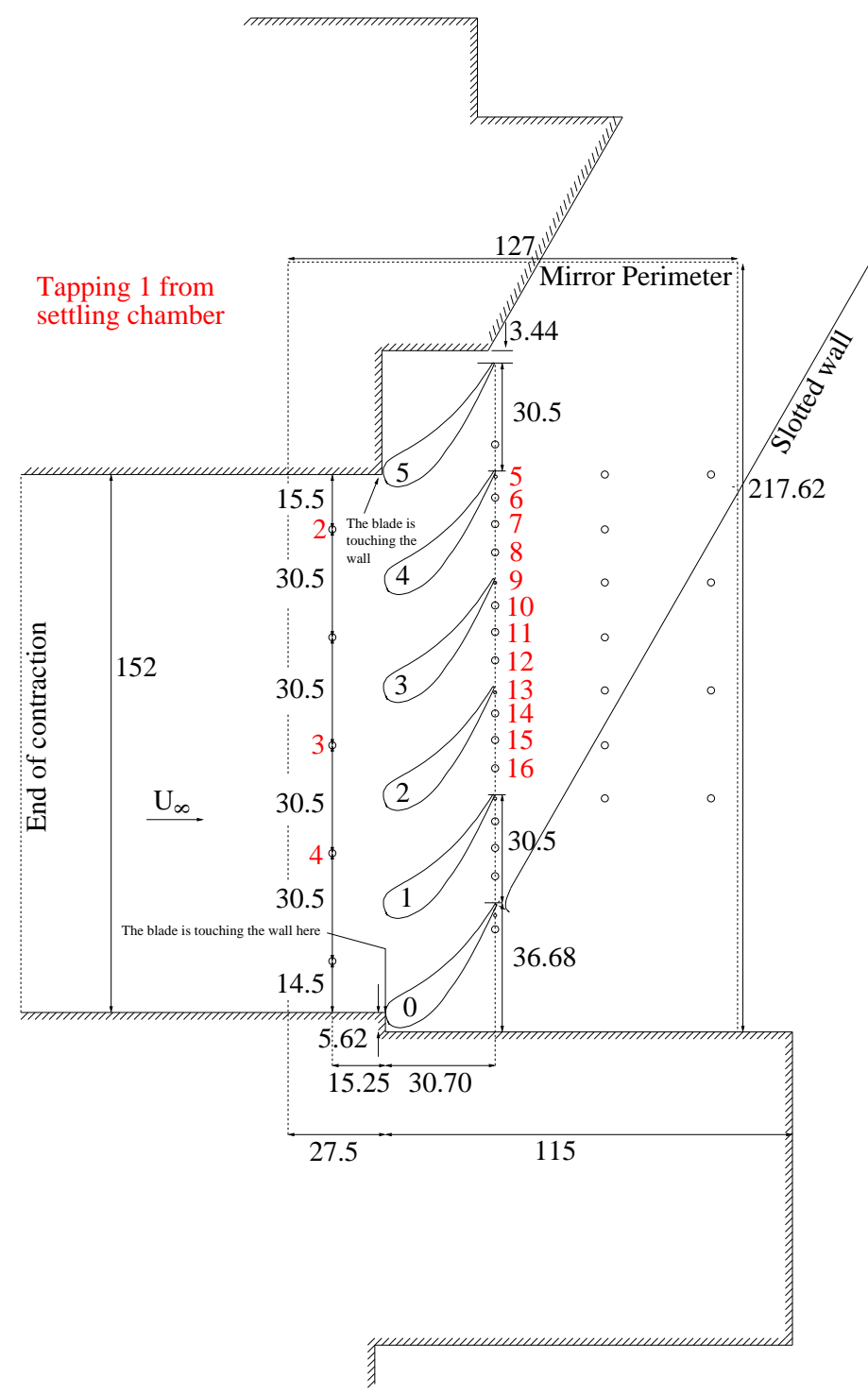

Fig. 1 Side view of the wind tunnel test section. All dimensions are in $\mathbf{m m}$.

allows the tailboard angle to be adjusted with respect to the cascade outflow direction. The trailing edge of the chassis is secured to the mirrored back wall and to the frame of the front schlieren glass by grooved $\mathrm{T}$ pieces, which allow the tailboard angle to be adjusted between tests. In the present work, tests have been conducted with a tailboard angle of $62^{\circ} \pm 0.083^{\circ}$ and of $64^{\circ} \pm 0.083^{\circ}$ to the inflow direction. The chassis has a $175 \mathrm{~mm} \times 42 \mathrm{~mm}$ open area at its centre. Overlapping this central area, the first tailboard shim has $4.7 \mathrm{~mm}$ wide laser cut streamwise slots staggered $3.7 \mathrm{~mm}$ apart, sided by two narrower $2.35 \mathrm{~mm}$ slots. This pattern, detailed in Fig. 2(b), gives a geometric porosity or void ratio of approximately $47 \%$ over the open area of the chassis. The second tailboard shim is $200 \mathrm{~mm} \times 50 \mathrm{~mm}$ in wetted area and has four evenly spaced

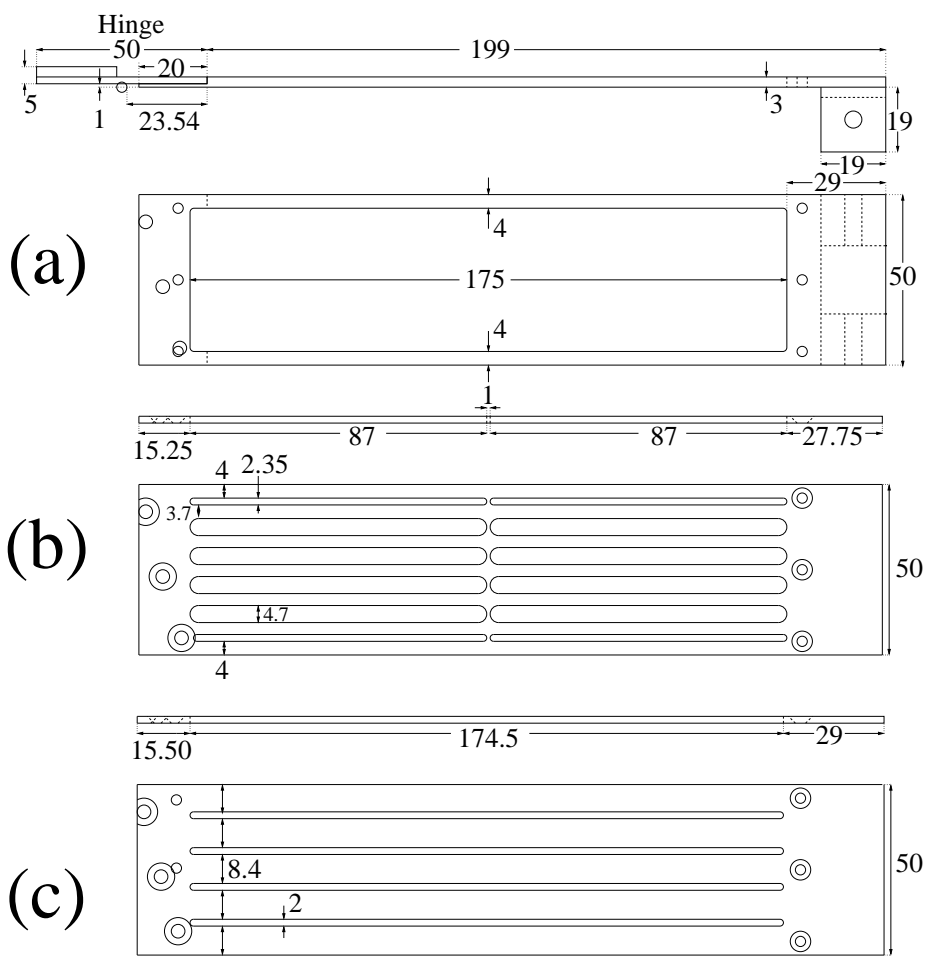

Fig. 2 Tailboards tested in the cascade tunnel: (a) Support chassis for slotted boards (b) and (c), (b) $47 \%$ void ratio board, (c) $15 \%$ void ratio board. All dimensions are in $\mathbf{m m}$.

$2 m m$ wide streamwise slots. This pattern, shown in Fig. 2(c), gives a void ratio of approximately $15 \%$. An aluminium in-fill has been cut and filed from a $3 \mathrm{~mm} \times 50 \mathrm{~mm} \times 2 \mathrm{~mm}$ thick shim to fill a small gap between the $15 \%$ void ratio tailboard leading edge and the suction side of the blade 0 trailing edge.

\section{Numerical Models}

Two different numerical models have been used to simulate the transonic cascade flow discharge. A three-dimensional RANS model was used to predict the flow around a single blade with pitchwise periodic boundaries. This prediction was used to study the discharge three-dimensionality and the effects of small-scale turbulence on the mean flow, in order to evaluate whether a simpler, two-dimensional, inviscid model could capture the main flow features of the discharge at a reduced computational cost. A two-dimensional Euler numerical method with a slotted wall boundary condition was then used to study the flow in the full test section and to determinate the tailboard settings that minimise wind tunnel end-wall interference in pitch.

\section{Pitchwise periodic flow models}

Pitchwise periodic flow predictions of the cascade discharge have been obtained with the in-house computational fluid 
dynamics software suite GENESIS at the ALSTOM Power Technology Centre. This three-dimensional (3D) numerical method is a dedicated turbomachinery design tool for non-reacting compressible flows. It uses a finite volume formulation and the SIMPLE implicit integration procedure by Patankar. ${ }^{10}$ The scheme solves the Reynolds Averaged Navier-Stokes (RANS) equations that govern the turbulent compressible cascade discharge flow. Turbulence closure is obtained by a $k-\epsilon$ two-layer model, in which the Wolfstain one-equation model is used in the near wall region. ${ }^{11}$ The near wall region is defined as the layer in which the wall-distance Reynolds number $R e_{y}=y \sqrt{k} / \nu<200$, where $y$ is the normal distance from the nearest wall, $k$ is the specific turbulent kinetic energy and $\nu$ is the kinematic viscosity.

In the finite-volume method, the inviscid fluxes are estimated at the unit cell boundaries using a third order QUICK scheme. Central differencing is used to determine the viscous fluxes. The computation is integrated implicitly with a Courant number of 100 . Under-relaxation of the pressure field residuals is used to enhance the scheme stability during the early stages of the computation with a typical underrelaxation factor of 0.5 . A multi-block cell vertex body fitted mesh discretises the computational domain, whose streamwise boundaries are set to match the inviscid model ones. Specifically, the inflow boundary is set 0.5 blade chords upstream of the profile leading edge and the outflow boundary is located 4.1 blade chords downstream of the trailing edge. Along the inflow boundary, profiles of constant total pressure, total temperature and Mach number are imposed. The ambient to total pressure ratio is 0.3728 , the total temperature is $300^{\circ} \mathrm{K}$ and the inflow is axial. At the outflow, a pitchwise uniform back pressure $p_{e}=107752 \mathrm{~N} / \mathrm{m}^{2}$ is imposed. This is slightly higher than the typical laboratory ambient pressure $p_{a}=101325 \mathrm{~N} / \mathrm{m}^{2}$ in the tests. This higher back pressure aims to account for the static pressure loss in the wind tunnel outlet section due to the absence of a diffuser. In the flow normal direction, the computational domain spans one blade pitch, which is 0.67 of the blade chord ${ }^{8}$ from the blade suction side to the blade pressure side. No-slip conditions are imposed along the pitchwise solid boundaries and pitchwise periodicity is assumed away from the walls.

The multi-block computational mesh structure used in the RANS simulations has an ' $\mathrm{H}$ ' curvilinear body fitted topology, as shown in Fig. 3. For clarity, Fig. 3 displays only one mesh point every four in the flow normal direction and one mesh point every two in the streamwise direction. This Cartesian mesh uses standard indexes $(i, j, k)$, with the origin at the bottom left hand corner on the front face of each mesh block, as indicated in Fig. 3. Index $i$ follows the blade pitch and index $j$ follows around the profile in the streamwise direction. In block 5 , the $(i=71)$ top block boundary follows around the blade trailing edge, from the pressure side to the suction side,

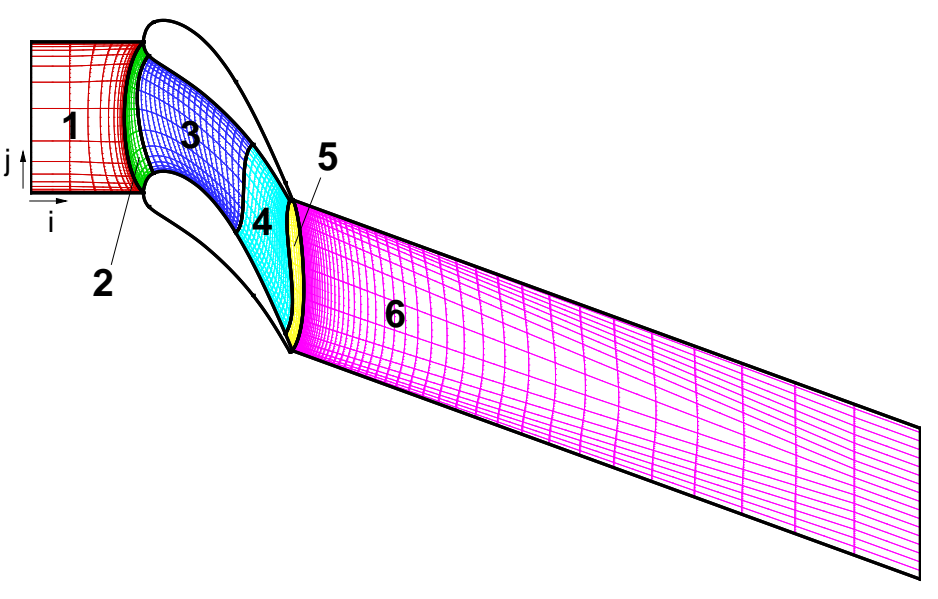

Fig. 3 Curvilinear $H$ mesh of the RANS model GENESIS. One in two mesh points is shown along $i$ and one in four along $j$.

\begin{tabular}{|l|c|c|c|c|c|}
\hline Block & $i_{\max }$ & $j_{\max }$ & $\begin{array}{c}i=1 \\
\text { stretch }\end{array}$ & $\begin{array}{c}i=i_{\max } \\
\text { stretch }\end{array}$ & $\begin{array}{c}j=1 \\
\text { stretch }\end{array}$ \\
\hline \hline 1 & 71 & 23 & 0.8454 & 0.8454 & 1.1309 \\
2 & 71 & 21 & 1.0554 & 1.0229 & 1.1349 \\
3 & 71 & 41 & 1.0224 & 1.0578 & 1.1201 \\
4 & 71 & 41 & 1.0000 & 0.9182 & 1.0933 \\
5 & 71 & 15 & 0.8229 & 0.9982 & 1.1234 \\
6 & 71 & 91 & 1.0569 & 1.0569 & 1.1346 \\
\hline
\end{tabular}

Table 1 Mesh parameters for the $2 \mathrm{D}$ cascade model.

to avoid placing a cusp in the base flow region. In block 6 , the pitchwise boundaries are set at $20^{\circ}$ to the cascade inflow direction. This orientation is a compromise between aligning the boundaries along the $\sim 63^{\circ}$ discharge direction, to best resolve trailing edge wakes, and using boundaries parallel to the inflow, to best resolve trailing edge shocks. These compressible flow features are further described later on. Each edge in the computational mesh is stretched with constant geometric expansion ratios, to cluster the computational grid in the neighbourhood of solid boundaries. In pitch, the mesh is stretched at both ends, except at the outflow boundary, where it is pitchwise uniform. Table 1 details the mesh parameters used in this study. The grid blocks are numbered as in Fig. 3.

The 3D mesh uses the same pitchwise and streamwise domain discretisations of the $2 \mathrm{D}$ model. In span, the $3 \mathrm{D}$ computational domain is $25 \mathrm{~mm}$ deep and models the flow area from the cascade mirrored back wall to the cascade wind tunnel mid-span (Fig. 1). In the numerical model, a no-slip con- 
dition is imposed at the location of the back mirror and a symmetric flow condition is imposed at the other spanwise computational boundary. 71 mesh points are used in span. A geometric stretching factor of 1.075 clusters the grid in span close to the no-slip wall.

The computation is started from ambient zero flow conditions throughout the domain. A coarse solution is determined by integrating just the inviscid fluxes on a coarse mesh, obtained by considering one every five mesh points in each direction $i, j, k$. This solution is typically obtained over 1000 implicit integrations. The coarse solution is then linearly interpolated onto the full computational mesh detailed in Table 1. A further set of 800 implicit integrations is then completed that include the contributions from the viscous fluxes and the turbulence model. This results in a converged solution with a typical mass inflow-outflow error of less than $1 \%$.

Two-dimensional model of the full cascade with tailboard

A key objective of this study is to predict how a slotted wall can reduce spurious reflections from shock and expansion waves impinging against a cascade tunnel pitchwise boundary. Since such flow features will be shown to be essentially inviscid phenomena, a two-dimensional (2D) inviscid model is used to study these compressible effects.

This model is not expected to capture the growth of boundary layers around the cascade profiles nor of the trailing edge shear layers in the cascade discharge. While such viscous flow features are known to modify the discharge flow geometry to some extent, their influence upon establishing a pitchwise periodic outflow in a slotted wall cascade tunnel may be of secondary importance. This issue is explored in this paper by cross-validating the ALSTOM Euler model predictions against results from the 2D and 3D Reynolds Averaged Navier-Stokes (RANS) models.

The inviscid numerical method is a computational fluid dynamics tool developed and deployed at the University of Rome La Sapienza. This numerical scheme is described in Di Mascio $\&$ Favini $^{12}$ and in Rona et al. ${ }^{4}$ It is a two-dimensional second order accurate Godunov ${ }^{12}$ type method, derived from the general form of Essentially Non-Oscillatory (ENO) schemes. ${ }^{13}$ This multi-block numerical method uses a structured bodyfitted $\mathrm{C}$ mesh around each T2 blade profile, as shown in Fig. 4(a) by Mesh 1. Mesh 1 is replicated and stacked in pitch to model the geometry of a linear cascade. This stack is shown as Mesh 2 in Fig. 4(b). Further mesh parameters are given in Paciorri et al. ${ }^{6}$ and in Rona et al. ${ }^{7}$

The mesh stacking in Fig. 4(b) gives a rugged outflow boundary, highlighted in Fig. 4(b) by a dotted line. The streamwise distance between this rugged boundary and the blade passage outlet is variable, therefore an appropriate linearly variable back-pressure boundary condition was imposed along this staircase edge.

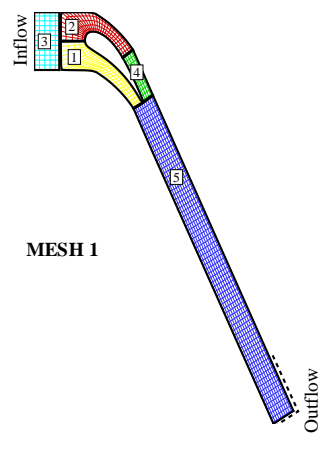

a)

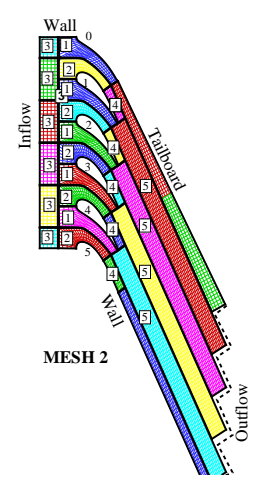

b)
Fig. 4 Meshes and boundary conditions. Paciorri et al. ${ }^{6}$ inviscid model.

A slotted tailboard model by Paciorri et al. ${ }^{6}$ is included in the numerical method. This model has been benchmarked ${ }^{6}$ at compressible regimes using a Mach 1.8 oblique shock test case by McLafferty. ${ }^{14}$

\section{Results}

\section{Pitchwise periodic numerical flow predictions}

Pitchwise periodic numerical boundaries allow to predict the flow through an ideal cascade with a very large number of blades. This gives a target flow field to compare against corresponding results from wind tunnel tests with a limited number of blades, in which a slotted tailboard is used to preserve the flow periodicity in pitch.

Numerical solutions with pitchwise periodic boundaries have been computed with the $2 \mathrm{D}$ Euler, the $2 \mathrm{D}$ and the $3 \mathrm{D}$ RANS models at the ALSTOM Power Technology Centre. Predictions of the isentropic Mach number along the blade profile, referenced to the inflow stagnation conditions, are reported in Figure 5. The isentropic Mach number is derived from the blade surface pressure and is plotted in Figure 5 against the chord fraction $s / c$ from the blade leading edge, $s / c=0$, to its trailing edge, $s / c=1$.

Using a turbulence closure model induces no appreciable change to the blade isentropic Mach number distribution, with respect to the ALSTOM Euler prediction. The prediction from the 2D $k-\epsilon$ model and from the ALSTOM inviscid method overlap.

The 3D $k-\epsilon$ prediction reported in Fig. 5 with a dashdouble dot line shows the isentropic Mach number distribution at the cascade mid-span, on the CFD symmetric boundary plane. This prediction overlaps with the $2 \mathrm{D} k-\epsilon$ and the 2D Euler model $M_{i}$ distributions over the blade pressure side and on the suction side upstream of $s / c=0.6$. The local maximum in $M_{i}$ at $s / c \simeq 0.62$ is slightly higher than the inviscid model prediction, indicating a slight over-expansion downstream of the sonic throat in the 3D geometry. These slight 


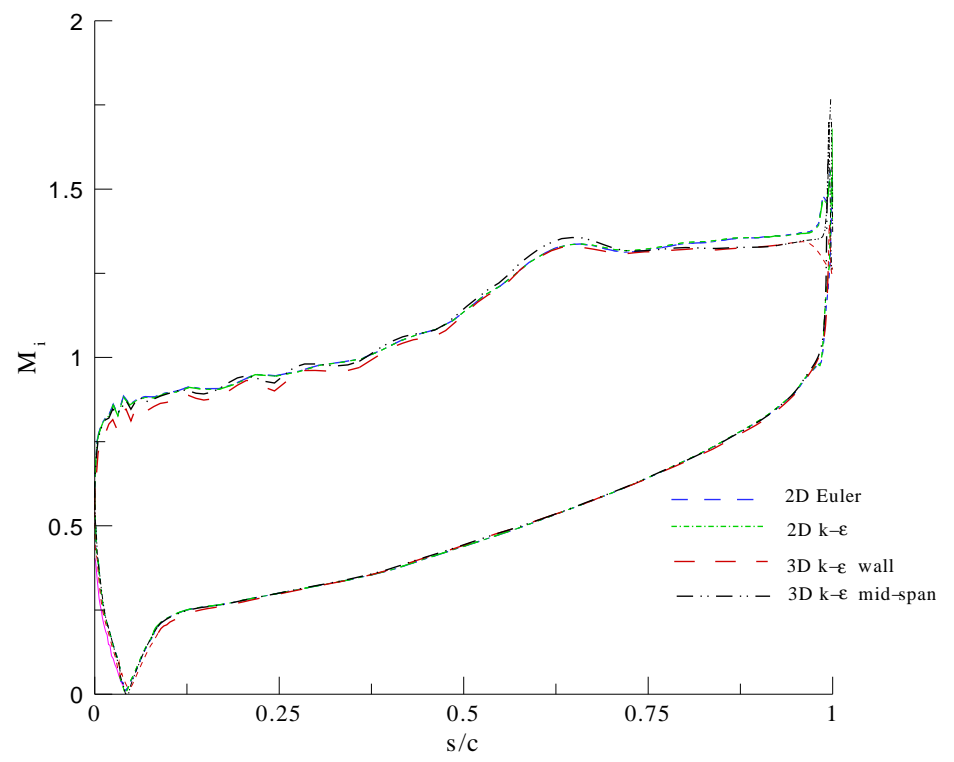

Fig. 5 Predicted isentropic Mach number distribution along the blade chord with pitchwise periodic boundaries.

differences in $M_{i}$ indicate that the $50 \mathrm{~mm}$ wind tunnel cascade span may be sufficient to devoid the mid-span isentropic Mach number distribution from any significant spanwise end wall interference effects.

On the wind tunnel end-wall, the isentropic Mach number distribution from the 3D $k-\epsilon$ model follows essentially the same trend as the mid-span prediction. On the blade pressure side, the favourable streamwise pressure gradient demotes the growth of the surface boundary layers, both on the end wall and on the blade surface, compared to the suction side. The thin boundary layer displacement thicknesses slightly alter the passage cross-sectional area seen by the equivalent inviscid flow, compared to the 2D Euler prediction, between $s / c=0$ and $s / c=0.25$. In the boundary layers, the dissipation of turbulent kinetic energy drains kinetic energy from the mean flow. This results in the pressure side $M_{i}$ prediction from the 2D $k-\epsilon$ model lying just below the inviscid model one in the range $0.25 \leq s / c \leq 0.75$. A similar trend was observed by Medic \& Durbin ${ }^{11}$ in relation to a VKI transonic cascade. ${ }^{15}$ Over the suction side, the secondary flow effects due to the presence of the end-wall and suction side boundary layers result in a similar reduction in isentropic Mach number in the range $0 \leq s / c \leq 0.7$. This reduction is slightly more marked in the neighbourhood $s / c \sim 0.62$. At this location, the flow re-compresses due to a fish-tail shock from the neighbouring blade impinging on the suction side. In this model flow, the presence of neighbouring blades is modelled the pitchwise periodic boundary conditions. This local flow re-compression promotes the local boundary layer growth through its adverse streamwise pressure gradient, enhancing the flow kinetic energy drain by turbulent kinetic energy dissipation in the boundary layers.

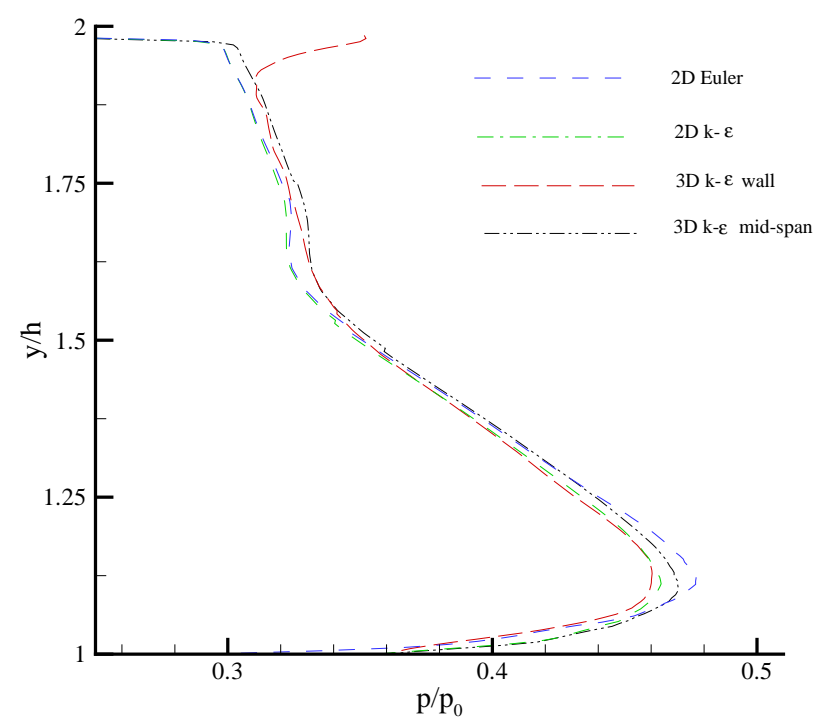

Fig. 6 Predicted normalised static pressure distribution at the passage exit. Pitchwise periodic boundaries.

Figure 6 compares the predictions of static pressure $p$ along the passage exit, obtained from the $2 \mathrm{D}$ inviscid model with those from the 2D RANS and 3D RANS computations. The results are normalised by the inlet stagnation pressure $p_{0}$. These normalised pressure distributions cover one blade pitch $h$ in the positive $y$ direction, where $y$ is the pitch coordinate, taken positive from the trailing edge of blade 0 to the trailing edge of blade 5 (Fig. 1). They are drawn from the exit plane of the blade passage and are evaluated on the respective curvilinear computational domains by linear interpolation with the closest out of plane pressure data.

Just like in the $M_{i}$ distributions of Fig. 5, there appears to be only minor differences between the exit static pressure distribution predicted by the $2 \mathrm{D}$ inviscid method and the one from the $2 \mathrm{D} k-\epsilon$ prediction. These two $p / p_{0}$ distributions in Fig. 6 overlap over most of the blade pitch, the inviscid distribution predicting a pressure maximum slightly further away from the blade pressure side. The $3 \mathrm{D} k-\epsilon$ prediction at the cascade mid-span lies close to the 2D Euler and 2D $k-\epsilon$ distributions across most of the blade pitch. Close to the suction side trailing edge, the 3D $k-\epsilon$ distribution is higher than the $2 \mathrm{D}$ predictions. This supports the inference from Fig. 5 with regards to the supersonic discharge being less expanded at the cascade exit in the $3 \mathrm{D}$ model than in the 2D simulations, due to the enhanced growth of the boundary layers over the shock-induced adverse pressure gradient along the suction side trailing edge.

The pressure distribution on the cascade end-wall is shown by a long dashed line in Fig. 6. This distribution follows reasonably well the $3 \mathrm{D} k-\epsilon$ prediction at the cascade mid-span, 
indicating that the pressure field is reasonably uniform spanwise, except in the small region of the suction side trailing edge base flow. Here, the confluence of the suction and pressure side boundary layers into a trailing edge shear layer is affected by the end-wall boundary layer. This probably gives a more stagnant flow in the base region that results in a higher static pressure at the end-wall than at the cascade mid-span.

The comparison among the numerical predictions presented so far has shown that the periodic flow results from the inviscid 2D numerical model produces blade loading and exit passage pressure distributions similar to the ones from to the more computationally expensive 2D RANS and 3D RANS simulations. The dominant two-dimensionality of the static pressure field at the cascade exit plane justifies a direct comparison between end-wall pressure measurements and 2D predictions of static pressure, presented later on. Furthermore, the $2 \mathrm{D}$ inviscid model predictions can be used, with some confidence, as a point of reference to steer the design of perforated tailboards, towards improving the pitchwise periodicity in the cascade wind tunnel.

\section{Secondary flows}

The experimental flow visualisations of Figs. 7, 8a and 9a show evidence of secondary flows developing over the wind tunnel mirror end-wall. A growing boundary layer develops over this flat surface. As this boundary layer approaches the leading edge of the blades, it experiences an adverse pressure gradient in the neighbourhood of the leading edge stagnation point. This pressure gradient causes the boundary layer to separate, rolling up a near-wall horse-shoe vortex just upstream of the leading edge. The vortex core wraps around the leading edge, deploying vortex filaments on both suction and pressure sides. These filaments run along the profile in the downstream direction. The vortex centreline is visualised in Figs. 7, 8a and 9a as a dark ' $\mathrm{C}$ ' shaped streak around the leading edge, stretching in the downstream direction. The suction side branch remains clearly visible in Fig. 7 for up to about two chords downstream of the trailing edge. In Fig. 8a, this branch of the horse-shoe vortex is more faint in this visualisation and it is best observed around blade 3 .

The extent to which these secondary flows disrupt the cascade discharge two-dimensionality cannot be readily assessed from the experimental schlieren visualisations, as these give an integrated view of the flow density gradients across the test section span. The predicted pressure distributions at the cascade exit plane in Fig. 6 suggest that secondary flow effects on the pressure field are very modest. An improved assessment of the cascade flow three-dimensionality is provided by the 3D $k-\epsilon$ model predictions of the near-wall spanwise velocity, shown in Fig. 10. In Fig. 10, several spanwise cross-sections of the pitchwise periodic flow are stacked in pitch, for ease of comparison. Near the no-slip end-wall, at $z / c=0.002$, an outer rim of positive spanwise velocity encloses a rim of nega-

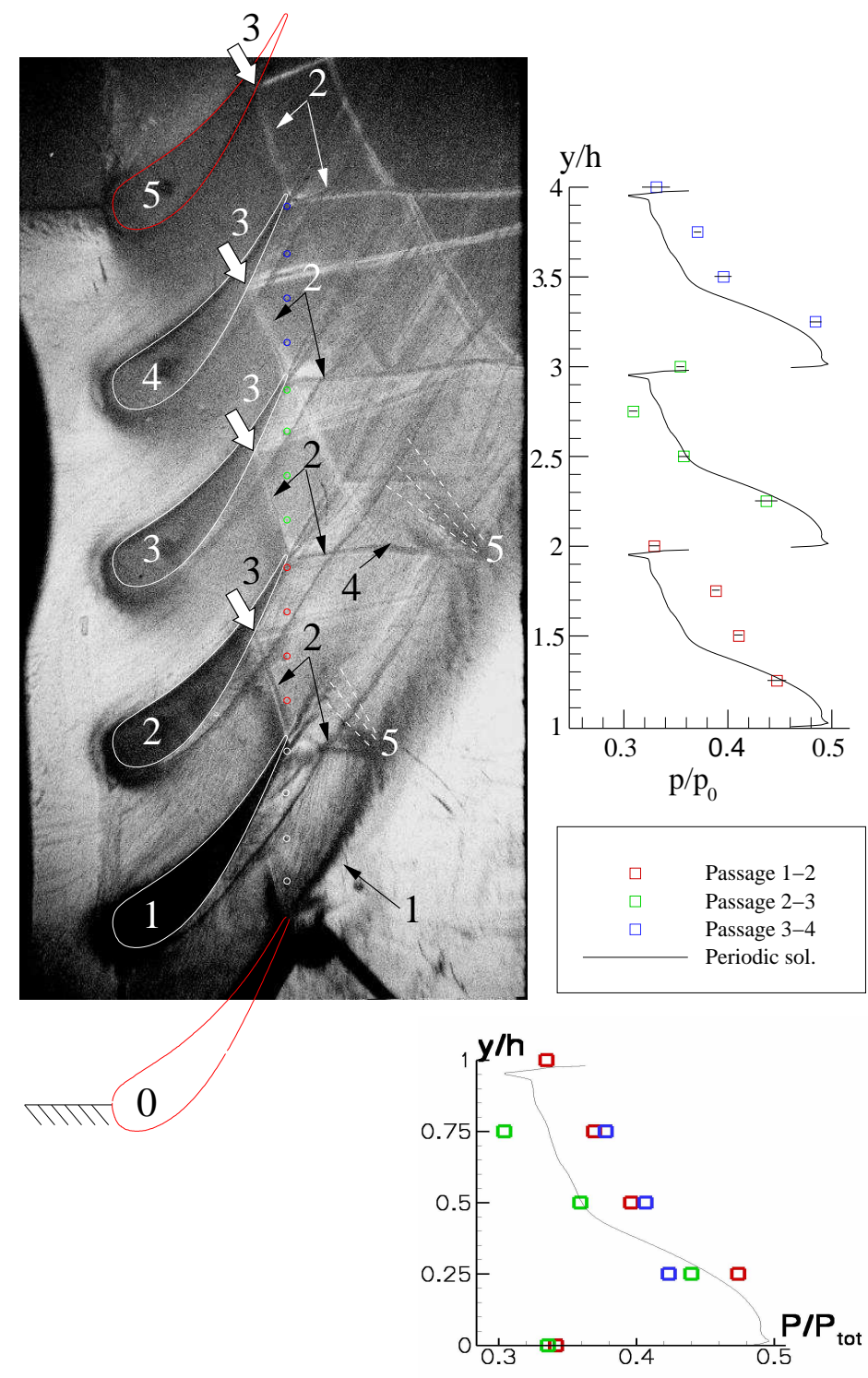

Fig. 7 Experimental schlieren visualisation of the cascade flow discharge without a slotted wall.

tive spanwise velocity, around the blade trailing edge. These rims are respectively the upwash and the downwash of the leading-edge horse-shoe vortex, which is captured by the numerical method. As the vortex stretches downstream along the blade surface, it grows in size by fluid entrainment. This results in the displacement of the vortex core away from the solid end-wall. This process is indicated by the gradual spanwise rise and downstream shift of the region of highest upwash along the suction surface at increasing $z / c$. The spanwise location where the largest upwash is reached should correlate reasonably well with the vortex core height above the endwall, marking the streamwise rise of the vortex core.

Figure 10 also highlights a significant interaction of a fish- 

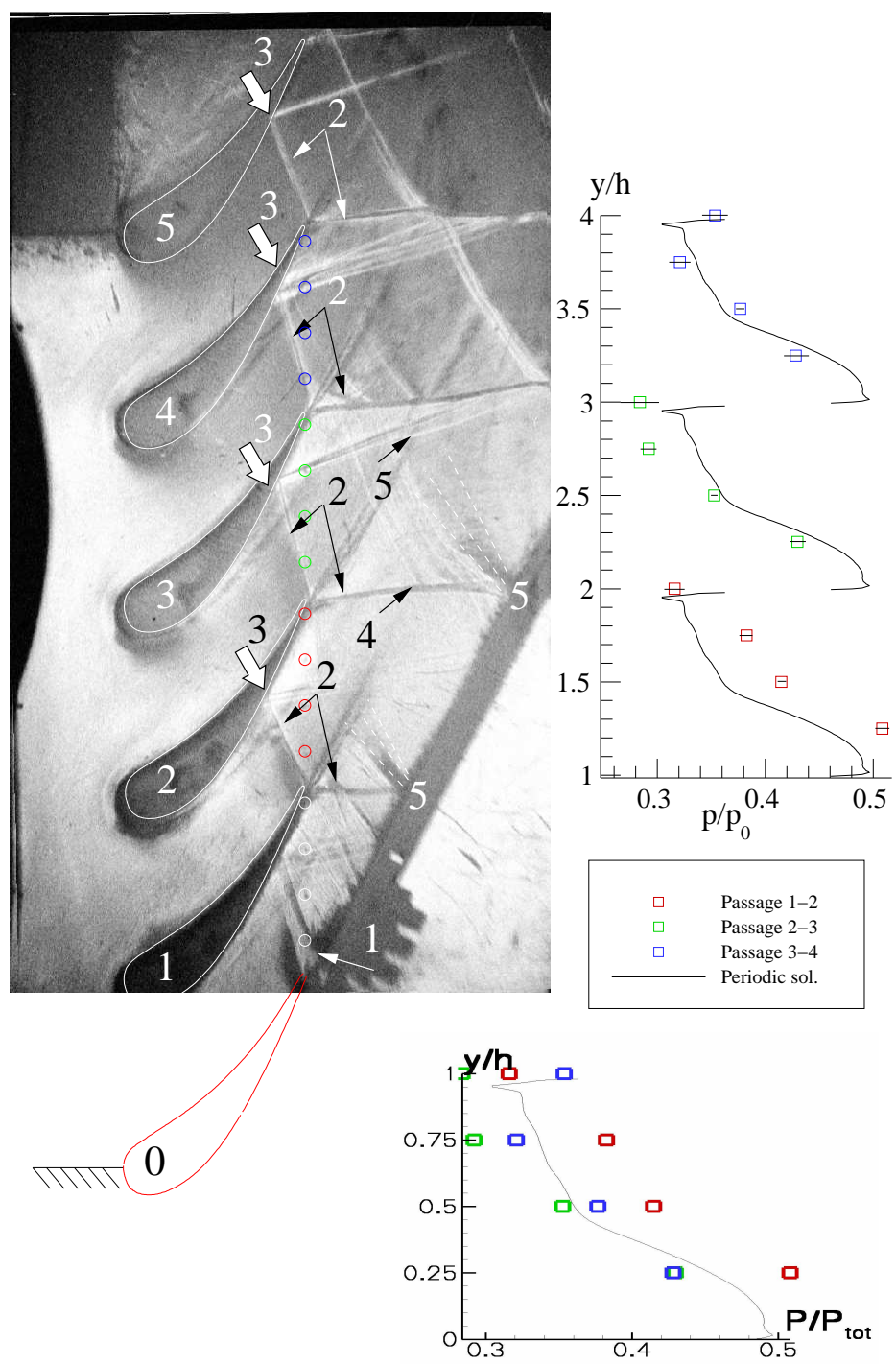

a) Experimental
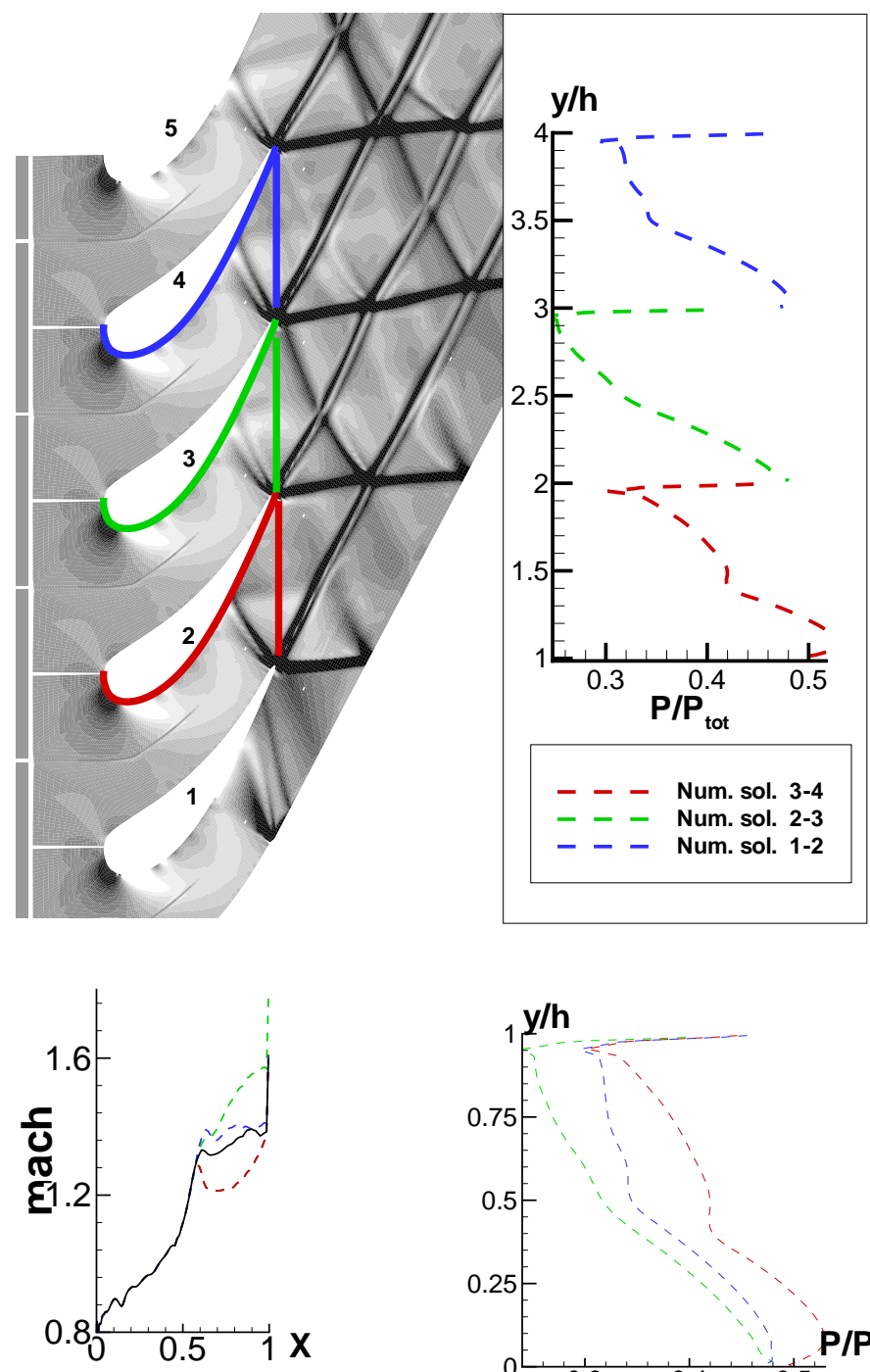

b) Numerical

Fig. 8 Results of the cascade flow discharge with a $50 \%$ void ratio tailboard.

tail trailing edge shock with the end-wall boundary layer. The shock imposes an adverse pressure gradient on the near-wall flow. This is expected to induce a rapid growth in the local boundary layer displacement thickness that lifts the flow away from the end-wall. Further three-dimensional mixing also occurs along the trailing edge near-wall shear layer.

Further away from the end-wall, towards the cascade midspan, the secondary flow effects observed in Fig. 10 weaken as the spanwise distance increases. By $z / c=0.119$ there is no evidence of any significant horse-shoe vortex induced spanwise velocity left in the domain. This confirms that the horse-shoe vortex is a secondary flow that is confined to the wall boundary layer. Further away from the wall, the shock induced upwash on the end-wall boundary layer flow also weakens, together with the three-dimensional shear- layer mixing. On approach to the cascade mid-span, at $z / c=0.355$ and $z / c=0.511$, the results of Fig. 10 indicate that the spanwise velocity is $<0.5 \%$ of the $410 \mathrm{~m} / \mathrm{s}$ predicted maximum streamwise velocity at mid-span on the discharge plane. This indicates that the time-averaged flow is essentially two-dimensional at the mid-span. This result supports the selection of the $50 \mathrm{~mm}$ wind tunnel cascade working section cross-section as being of adequate span to measure the two-dimensional characteristics of the $\mathrm{T} 2$ profile at the given inflow conditions. It also confirms that a $2 \mathrm{D}$ numerical modelling approach to study the cascade periodicity in this flow is appropriate. 

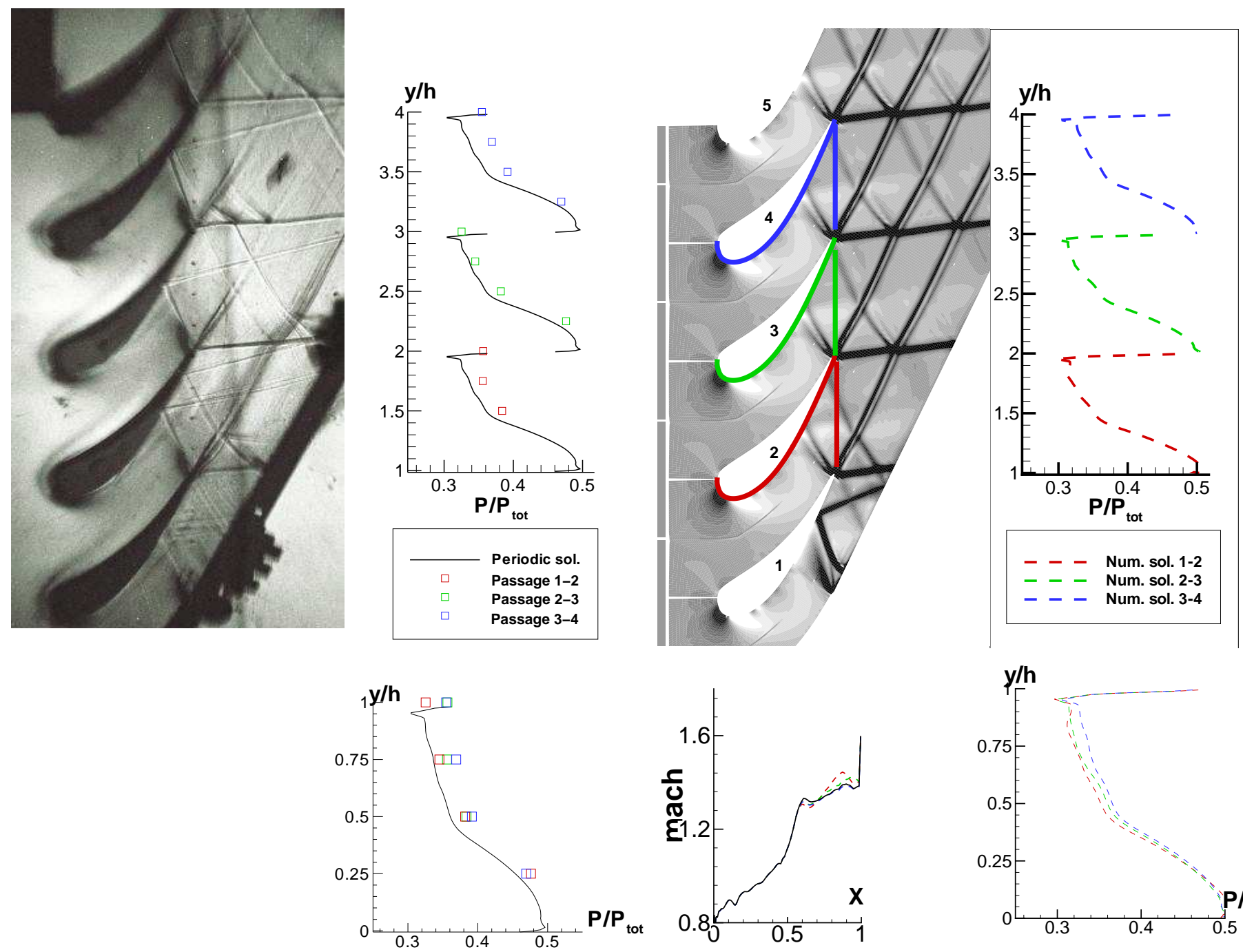

a) Experimental
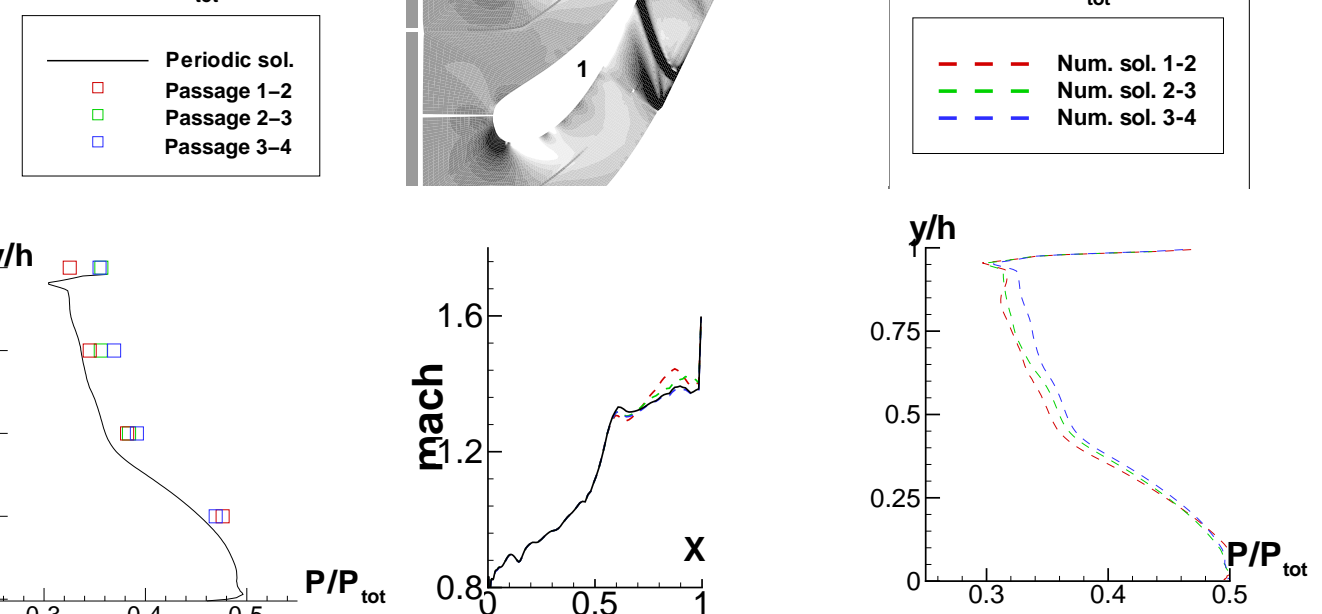

b) Numerical

Fig. 9 Results of cascade flow discharge with a $15 \%$ void ratio tailboard.

\section{Baseline flow without tailboard}

Figure 7 shows a selected schlieren record of the linear nozzle cascade tested at an isentropic discharge Mach number $M_{d}=1.27$. Downstream of the blade 0 trailing edge, a thick shear layer is formed by the mixing of the high speed flow from the tunnel discharge with the surrounding air in the laboratory. This shear layer is significantly thicker compared to the mixing regions downstream of the trailing edges of the other blades in the cascade. The shear layers are not pitchwise periodic and produce visible differences in the flow discharged from different cascade passages. A different discharge from each passage results in a non-pitchwise pressure distribution, as shown in the wall pressure measurements of Fig. 7 to the right of the schlieren image. The square symbols show results from synchronous pressure measurements at $M_{d}=1.27$. The continuous line is the pitchwise periodic numerical predictions and serves as reference to compare the experimental pressure data among the passages. There is significant passage to passage variation in these pressure records. This variation is highlighted in the right bottom frame of Fig. 7, where pressure measurements across passages 2,3 and 4 are overlaid. The source of this non-pitchwise flow discharge has been further investigated in Paciorri et al. ${ }^{6}$ and in Rona et al. ${ }^{7}$

\section{Slotted tailboard with $47 \%$ void ratio}

To attempt to improve the pitchwise periodicity of the cascade flow, the test section was fitted with the $47 \%$ void ratio tailboard of Fig. 2(b), as shown in Fig 1. The cascade was tested at the same isentropic Mach number $M_{d}=1.27$ as the baseline flow and the tailboard angle was set at $62^{\circ} \pm 0.083^{\circ}$ to the cascade inflow direction. The $62^{\circ}$ tailboard pitch is lower than the $63.4^{\circ}$ blade metal angle, determined from the profile geometry. ${ }^{8}$ This setting was chosen to allow for the 


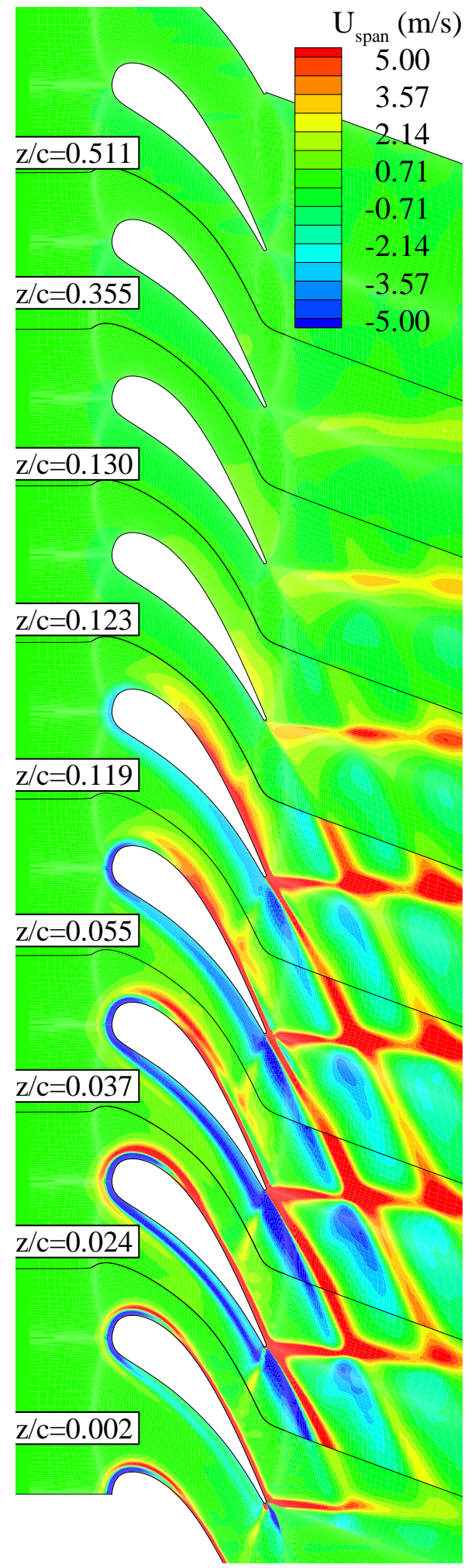

Fig. 10 Numerical results of cascade flow discharge with pitchwise periodic boundaries at different spanwise crosssections $z / c$. Colour map of spanwise velocity $(\mathrm{m} / \mathrm{s})$. growth of the boundary layer displacement thickness $\delta^{*}$ along the board. Specifically, $\delta^{*}$ displaces the discharge away from the boundary, turning it inwards, towards the central passages. This increases the effective tailboard pitch, which, at $M_{d}=1.27$, becomes close to the $63^{\circ}$ flow discharge. The selected schlieren record of Fig. 8a indicates that the presence of the tailboard has eliminated the thick shear layer downstream of blade 0 and has attenuated a number of shock-shear layer interactions both downstream of blade 0 , in passage 1 , and in the discharge from the other passages. The variation in wall static pressure from one passage to the next is still significant, as shown in the right bottom frame of Fig. 8a, indicating that there is scope to further refine the slotted wall geometry and to improve the pitchwise periodicity of the linear cascade flow.

The numerical method of Paciorri et al. ${ }^{6}$ has provided further insight into the working of a $50 \%$ void ratio board in this linear cascade tunnel. Figure 8 presents a numerical schlieren visualisation from the steady flow predicted by this numerical method. There is an appreciable agreement between the predicted wave pattern and the one recorded in experiment, presented in Fig. 8. The pressure differences among the passages, predicted by the numerical model, are shown in the right bottom frame of Fig. 8b. These are in good agreement with the experimental passage-to-passage pressure variation, shown in the left bottom frame of Fig. 8a. Numerical predictions and measurements indicate that, at the selected tailboard geometry and test conditions, the exit flow in the second passage has a static wall pressure above the periodic flow prediction and it is thus comparatively underexpanded. Conversely, the flow exiting from the third passage has a pressure distribution lower than the periodic prediction and the flow is relatively over-expanded. The pressure distribution across the fourth passage is predicted to lie close to the periodic flow model one and this is confirmed by the measurements.

Figure 8 shows the predicted isentropic surface Mach number distribution on the suction side of blades 2 to 4 (dashed lines) and compares it against the periodic flow prediction (continuous black line). The predictions overlap up to 0.6 chords downstream of the blade leading edges. Further downstream, significant differences among the predictions over blades 2 to 4 indicate differences in their suction side surface pressure, which results in a non-uniform pitchwise blade loading.

\section{Slotted tailboard with $15 \%$ void ratio}

Further numerical tests have been conducted to identify a slotted tailboard configuration that improves the periodicity in the cascade flow. These have led to a cascade flow model with a $14.3 \%$ void ratio board set at $64^{\circ}$ to the inflow direction. ${ }^{6}$ Figure 9 shows the experimental and the numerical schlieren visualisation from this configuration together with the respective pressure distributions. The normalised exit 
pressure predictions from passages 2 to 4 are reported to the right of the schlieren image. The corresponding suction side isentropic Mach number distribution is displayed below the model cascade. The predictions in Fig. 9b highlight improvements in the periodicity of the wall pressure distribution along the cascade trailing edge line with respect to Fig. 8, as indicated by the dashed lines being closely clustered together in Fig. 9b. This tailboard arrangement also predicts isentropic Mach number distributions on the blades suction side that are very close to one another, suggesting a more uniform blade loading throughout the cascade.

Wind tunnel tests with this tailboard configuration show a general improvement in the pitchwise periodicity of the passage exit pressure distributions respect to the numerical predictions. The experimental data are superimposed in Fig. 9a by square symbols $(\square)$. The data presented is derived from an ensemble of $35 \times 16$ measurements over three working days. In these tests, the ambient to reservoir pressure ratio was fluctuating about the design test point of $p_{a} / p_{0}=0.3728$, therefore the recorded pressure data were linearly interpolated in time at the $p_{a} / p_{0}=0.3728$ crossings. An ensemble of 35 acquisitions from each instrumented tapping was obtained at this design pressure ratio. Figure 9 displays at each location the ensemble mean by the square symbol and the two-sided standard deviation by the error bar. In Fig. 9, the largest standard deviation is $\leq 0.83 \%$ of the ensemble mean, indicating a good consistency in the measurements through the ensemble. This modest data scatter is in line with the measured uncertainties in Fig. 7 and in Fig. 8.

The best agreement between the measurements and the numerical predictions is obtained at the exit of the third passage, where the measurements and the predictions with tailboard lie close to each other, on either side of the pitchwise periodic numerical solution. Passages 2 and 4 are comparatively under-expanded with respect to the third passage but their pressure distributions are more similar to each other than the ones from passages 2 and 4 in Fig. 8, suggesting a tangible improvement in the flow periodicity with respect to the $50 \%$ void ratio board results.

\section{Periodicity error}

The different degree of pitchwise periodicity displayed by the cascade tests with and without a tailboard has been qualitatively observed in the previous sections. A more quantitative assessment of the tailboard performance in restoring the cascade pitchwise periodicity requires the definition of a periodicity error.

In an ideal pitchwise periodic cascade test, the measured end-wall pressure should repeat at the corresponding pitchwise coordinates $y$ over successive passages, so that, for instance, $p(1.50 h)=p(2.50 h)=p(3.50 h)$. The periodicity

\begin{tabular}{|l|c|c|}
\hline Test case & $\bar{E}$ experiment & $\bar{E}$ simulation \\
\hline No tailboard & 0.0939 & 0.0677 \\
$50 \%$ tailboard & 0.1019 & 0.1689 \\
$15 \%$ tailboard & 0.0218 & 0.0237 \\
\hline
\end{tabular}

Table 2 Summary of measured cascade periodicity error with different tailboard configurations.

pressure scatter at $50 \%$ blade pitch can be defined as

$$
\begin{aligned}
\Delta p_{50 \%}= & (|p(1.50 h)-p(2.50 h)|+ \\
& |p(1.50 h)-p(3.50 h)|+ \\
& |p(2.50 h)-p(3.50 h)|) / 3
\end{aligned}
$$

and similarly for other fractions of the blade pitch. The pitch average periodicity error is then obtained by integrating along the pitch with Simpson's rule and dividing the result by $h$. For the experimental data, this gives

$$
\bar{E}=\frac{1}{2}\left(\frac{1}{3} \Delta p_{25 \%}+\frac{4}{3} \Delta p_{50 \%}+\frac{1}{3} \Delta p_{75 \%}\right) / \bar{p}
$$

where $\bar{p}$ is a reference averaged exit pressure derived from the pitchwise periodic numerical solution of Paciorri et al. ${ }^{6}$ This is defined as: ${ }^{6}$

$$
\bar{p}=\frac{1}{0.5 h} \int_{0.25 h}^{0.75 h} p(y) d y
$$

Table 2 summarises pitch-averaged periodicity errors obtained by experiment and simulation in the three test cases presented in this paper. The results in Table 2 confirm that the $15 \%$ slotted board reduces the periodicity error with respect to the configuration without a tailboard, while the $50 \%$ board gives a larger error.

\section{Conclusions}

A joint numerical and experimental investigation has been conducted into the design of slotted walls for a transonic linear cascade wind tunnel. A 2D inviscid numerical model by Paciorri et al. ${ }^{6}$ was chosen to guide the tailboard design process and provide the means to assess the impact of any design improvement on the flow periodicity and on the periodicity of the blade to blade aerodynamic loads.

Two-dimensional inviscid predictions at Mach and Reynolds numbers representative of transonic turbine stator flows have been obtained and cross-validated against comparative 2D and 3D RANS predictions from the ALSTOM Power Technology Centre. A detailed study of the three-dimensional turbulent nozzle outflow concluded that the flow pitchwise periodicity is mainly driven by the predominantly $2 \mathrm{D}$ Euler pressure field in the test section. This study confirms that the 2D inviscid numerical approach is an adequate level of modelling to the study the pitchwise periodicity of the University of Leicester cascade tunnel. It also confirms that the 
$50 \mathrm{~mm}$ wind tunnel test section span is sufficient to give an essentially $2 \mathrm{D}$ flow at the mid-span.

Wind tunnel tests and numerical predictions at $M_{d}=1.27$ have directed the tailboard design process towards a $15 \%$ void ratio tailboard set at $64^{\circ}$ to the inflow direction, which was tested against the baseline free jet cascade configuration and a $47 \%$ void ratio slotted tailboard configuration from Rona et al. ${ }^{7}$ These tests confirmed that the $15 \%$ tailboard design significantly improves the periodicity of the cascade flow, reducing the periodicity error by $77 \%$ with respect to a free jet boundary, and by $79 \%$ with respect to the $47 \%$ void ratio tailboard. ${ }^{7}$

This work has validated an innovative numerical method to design pitchwise periodic boundaries for linear cascade wind tunnels. This constitutes a useful tool for turbomachinery blade designers who can rapid-prototype slotted tailboards to validate performance predictions of their blade profiles through cost-effective linear cascade tests.

\section{References}

${ }^{1}$ Goethert, B., Transonic wind tunnel testing, Pergamon Press, Oxford, UK, 1961 .

${ }^{2}$ Gostelow, J., Hobson, D., and Watson, P., "Preliminary evaluation of the influence of tailboard porosity on shock reflections in a supercritical nozzle cascade," Tech. Rep. TR 64, Department of Engineering, University of Cambridge, 1974.

${ }^{3}$ Rona, A. and Gostelow, J., "Split schlieren visualisation of a transonic linear cascade," Conference Paper F3037, Mar. 2001, In proceedings of the 3rd Pacific Symposium on Flow Visualisation and Image Processing.

${ }^{4}$ Rona, A., Paciorri, R., and Sabetta, F., "Experimental and Numerical Evaluation of Non-Reflecting Porous Tailboards for Transonic Cascade Wind Tunnels," Conference Paper ATI-CST-019/01, Mar. 2001, In proceedings of 4th European Conference on TURBOMACHINERY fluid Dynamics and Thermodynamics.

${ }^{5}$ Schreiber, H. A., Starken, H., and Steinert, W., "Linear Cascade - Transonic and Supersonic Cascades (including turbines)," AGARDograph 328, Aug. 1993.

${ }^{6}$ Paciorri, R., Sabetta, F., and Rona, A., "Wave reflection of porous walls: numerical modelling and application to transonic wind tunnels," Conference Paper 2002-1060, AIAA, January 2002, 40th AIAA Aerospace Sciences Meeting and Exhibit, Reno, NV.

${ }^{7}$ Rona, A., Gostelow, J., Paciorri, R., and Geron, M., "Wall intereference in the discharge flow in a linear cascade wind tunnel," Conference Paper 2003-0455, AIAA, Jan. 2003, 41st AIAA Aerospace Sciences Meeting and Exhibit, Reno, NV.

${ }^{8}$ Oldfield, M., Kiock, R., Holmes, A., and Graham, C., "Boundary layer studies on highly loaded cascades using heated thin films and a traversing probe," Transactions of the ASME Journal of Engineering for Power, Vol. 103, Jan. 1981, pp. 237-246.

${ }^{9}$ Holder, D. and North, R., Schlieren methods, Her Majesty's Stationary Office, London, UK, 1963.

${ }^{10}$ Patanker, S., Numerical heat transfer and fluid flow, Hemisphere, Washington D.C., USA, 1980.

${ }^{11}$ Medic, G. and Durbin, P., "Towards improved prediction of heat transfer on turbine blades," Transactions of the ASME Journal of Turbomachinery, Vol. 124, Apr. 2002, pp. 187-192.

${ }^{12}$ Di Mascio, A. and Favini, B., "A Two-Step Godunov-Type Scheme for the Euler Equation," Meccanica, Vol. 26, 1991, pp. 179-188.
${ }^{13}$ Harten, A., Engquist, B., Osher, S., and Chakravarthy, S., "Uniformly high order accurate essentially non-oscillatory schemes," $J$. Comp. Phys., Vol. 71, 1987, pp. 231-303.

${ }^{14}$ McLafferty, G. H., "Investigation of the Use of Slotted Walls to Cancel Shock Wave Reflection in Supersonic Flow," UAC Report R15127-3, October 1949.

${ }^{15}$ Camci, C. and Arts, T., "Short duration measurements and numerical simulation of heat transfer along the suction side of a film-cooled gas turbine blade," Transactions of the ASME Journal of Engineering for Power, Vol. 107, Apr. 1985, pp. 991-997. 